\title{
Analysis of Competing Topologies of Linear Induction Machines for High-Speed Material Transport Systems
}

\author{
Patrick L. Jansen, Long-Jang Li, and Robert D. Lorenz, Senior Member, IEEE
}

\begin{abstract}
This paper investigates longitudinal and transverse flux linear motor topologies for very high acceleration rate (3-4 g), and high speed $(120 \mathrm{~km} / \mathrm{h})$, material transport systems which have significant technical and economic potential in flexible manufacturing environments. The general topology under consideration is a double-sided, short-moving-secondary/ong-fixedprimary, linear induction motor (LIM). The transport system requirements place severe demands upon the machine topology. In addition to efficient thrust production, the linear machine must be capable of generating large unbalanced normal (i.e., lateral) forces in order to achieve electromagnetic (mechanically passive) steering of vehicles with both straight and curvilinear primaries. Air gap chording and short secondary effects are two additional design complications. Minimization of the primary leakage inductance is necessary not only to minimize inverter ratings but also to maximize the influence of the secondary state on the primary terminal properties for magnetic flux and position/velocity estimation.

The orientation of the magnetic flux (i.e., longitudinal versus transverse) has a significant impact upon the above concerns and is thus a major analytical focus of this paper. A hybrid system topology consisting of a longitudinal-flux orientation within straight sections and a transverse-flux orientation within curvilinear sections appears to offer the best of both topologies.

The intended contribution of this paper is a detailed comparison of LIM topologies leading to a better design selection for very high performance systems.
\end{abstract}

\section{INTRODUCTION}

A N ULTRA-FAST, linear induction motor-based system for the transfer of parts, tooling, raw materials, etc. in flexible manufacturing systems was proposed in [1] and [2]. Such systems have been shown to have a tremendous economic potential for even the most modern of current manufacturing facilities [2]. Their use would create a network in which material can be transferred within a manufacturing environment analogous to data transfer in computer networks. The linear motor based transport system is a continuous, closed structure with multiple loops with multiple access ports and

Paper IPCSD 95-10, approved by the Electric Machines Committee of the IEEE Industry Applications Society for presentation at the 1993 IEEE Industry Applications Society Annual Meeting, Toronto, Ontario, Canada, October 3-8. This work received support from the Wisconsin Electric Machines and Power Electronics Consortium (WEMPEC) of the University of Wisconsin, Madison, the National Science Foundation (NSF), and by Unico, Inc. in Franksville, Wisconsin. Manuscript released for publication January 20, 1995.

P. L. Jansen is with MK Rail Corporation, Boise, ID 83706 USA.

L. J. Li and R. D. Lorenz are with the Department of Electrical and Computer Engineering, University of Wisconsin, Madison, WI 53706 USA.

IEEE Log Number 9411117. multiple, independently controlled, transfer vehicles. The vehicles are steered electromagnetically to exit at desired access ports allowing interaction with the manufacturing/assembly equipment. Although design specifications are dependent upon the actual manufacturing application, in a typical system the vehicles might accelerate and decelerate at 3-4 $\mathrm{g}$ with maximum velocities of $60-120 \mathrm{~km} / \mathrm{h}(\approx 15-30 \mathrm{~m} / \mathrm{s})$ and payloads up to $25 \mathrm{~kg}$. Feasible radii of curvatures of one meter or less are anticipated. All modes of the vehicle operation are fully and continuously controlled, i.e., acceleration, deceleration, constant velocity, steering, tool/material transfer, etc. The system is well over an order of magnitude faster in acceleration and velocity than other systems currently in use or development [3], [4].

Reference [1] presented an overview of the technologies associated with the integrated vehicle/electromagnetic propulsion unit, which included linear motor topological design, vehicle dynamics, sensorless motion control, and distributed power conversion. A double-sided, short-movingsecondary/long-fixed-primary, linear induction motor (LIM) was selected for the machine topology. To avoid the reliability problems associated with sensors and transducers in the anticipated harsh operating environments, all machine states, e.g., position, velocity, and flux, must be estimated via observers with inputs from the machine terminal properties only, i.e., the primary voltages and currents.

Rather than levitating the vehicles, the electromagnetic forces that are normal to the thrust forces are used to steer the vehicles and to counteract centrifugal forces when traveling along a curved section. Thus no moving mechanical elements are required for steering. The double-sided topology with independently controlled primaries is essential for normal force generation and control. Ideally, via appropriate feedback control, the net normal forces acting on the vehicles would cancel such that the vehicles/secondaries are suspended between primaries. A more realistic goal is to significantly reduce the loading on the guide wheels and guideway structure

With a moving-secondary topology, the vehicle can be entirely passive. No power or control cables or connections to the vehicles are required, thereby permitting low mass, mechanically robust vehicles. Furthermore, the short-secondary topology does not suffer from dynamic end-effects [5] which severely degrade high-speed performance in long-secondary LIM's. 
The induction machine was chosen over permanent magnet (PM) machines due to its inherent mechanical robustness and field weakening capability. Both the exposed permanent field and the nonstructural properties of the PM are major liabilities in harsh environments. The induction machine secondary can be constructed of a cast aluminum cage surrounding steel laminations, the cast aluminum providing both a path for induced currents and structural integrity. Guide wheel, payload, and joint supports can be cast integral with the cage yielding an extremely robust vehicle.

The relatively uncommon transverse-flux topology (TFLIM) was shown to offer certain advantages (and some significant drawbacks) over the longitudinal-flux topology (LFLIM). A one-meter-long $E$-core TFLIM capable of over 3-g acceleration was constructed (details in [1]) to evaluate both performance and construction aspects.

This paper explores the detailed design attributes of the two competing topologies with respect to the unique constraints of the transport system, and ultimately proposes a hybrid system topology. The purpose of the paper is to develop a thorough understanding of the important design issues, much of which is founded on first hand design calculations and lab experience.

\section{COMmon Design Constraints}

Design issues and constraints that are common to both the LFLIM and the TFLIM with respect to the transport system are discussed in this section.

\section{A. Normal Force Generation and Control}

A vehicle traveling at $15 \mathrm{~m} / \mathrm{s}$ (about one-half speed) along a curvilinear primary with a one-meter radius of curvature will experience a centrifugal acceleration of $23 \mathrm{~g}\left(225 \mathrm{~m} / \mathrm{s}^{2}\right)$. This is nearly an order of magnitude higher than the maximum linear acceleration of this system. The machine must therefore be capable of generating electromagnetic normal forces at least an order of magnitude greater than the thrust forces. Since this ratio is common in conventional rotating induction motors with laminated squirrel cage rotors, a similar laminated cage construction permitting a high magnetic loading is well suited for the LIM secondary.

LIM's with solid aluminum secondaries operate with a relatively low magnetic loading and generate net repulsive normal forces, but these forces tend to be of the same magnitude as the thrust forces [6]. Furthermore, LIM's of low magnetic loading require longer pole pitch to air gap ratios for comparable quality [7]. As will be addressed, long pole pitches are not compatible with other design constraints.

With a high magnetic loading, the normal forces are predominantly attractive, being proportional to the square of the air gap flux density. The ability to manipulate both the normal force magnitude and its corresponding moment is a necessity during steering. Thus the spatial distribution of the air gap flux is of ultimate concern. Decoupled thrust and flux control is achievable via the direct field orientation approaches in [8].

\section{B. Magnetizing/Primary Leakage Inductance Ratio}

To achieve zero net normal force acting on the secondary, the air gap flux must be controlled such that it is equal on both sides. Exciting the two primaries with the same voltage (as via a parallel connection) will force the primary flux to be equal on both sides. If the primary leakage inductance was small relative to the magnetizing inductance, the air gap flux would then be forced nearly equal. Unfortunately, a short-secondary long-primary topology inherently has a relatively high primary leakage to magnetizing inductance ratio. In which case, knowledge of the primary leakage inductance is required to calculate and control the air gap flux. The parameter sensitivity analysis with respect to rotor flux estimation presented in [8] can be similarly applied to air gap flux estimation.

As with flux estimation, the robustness and accuracy of the vehicle position and velocity estimation hinges on a high magnetizing to leakage inductance ratio [9]. Since the primary leakage inductance can be estimated with only limited accuracy, its minimization with respect to the magnetizing inductance is a major design objective.

\section{Acceleration and Velocity}

For mechanical support reasons, the payload mass is constrained to be small relative to the secondary (and vehicle) mass. Thus the demand for high acceleration necessitates minimizing the moving secondary mass, i.e., minimizing the secondary core material. Furthermore, the normal force required for steering is also directly proportional to the vehicle mass.

In this and later sections, the optimal pole pitch is a major design issue. In this case, it relates excitation frequency to velocity. Assuming a maximum excitation frequency of 180 $\mathrm{Hz}$ at a synchronous velocity of $30 \mathrm{~m} / \mathrm{s}$, the required pole pitch is no less than $0.083 \mathrm{~m}$. For smaller pitches, the excitation frequency must increase, and primary core loss will also increase. The core loss is a significant consideration for the long-primary topology.

\section{Static End Effects}

Although dynamic end effects are eliminated with the shortsecondary topology, unbalances in either the secondary or primary phase-winding impedances will still introduce static end effects [5]. The impedance unbalances can be directly linked to asymmetries or discontinuities in the magnetic and electric circuits, such as the finite length cage associated with the short-secondary topology. Static end effects ultimately lead to a reduced overall machine performance due to poor utilization of core and/or conductor material, increased losses, and generally undesirable pulsations in the thrust, flux, and currents.

\section{E. Modular Construction}

In the event of primary winding or guideway failure, a modular construction consisting of interchangeable primary/guideway segments (or "units") and distributed controllers is preferred to minimize system downtime. The primary laminations and windings must therefore be of finite length segments, introducing the potential susceptibility to additional undesirable end effects in the primary. 


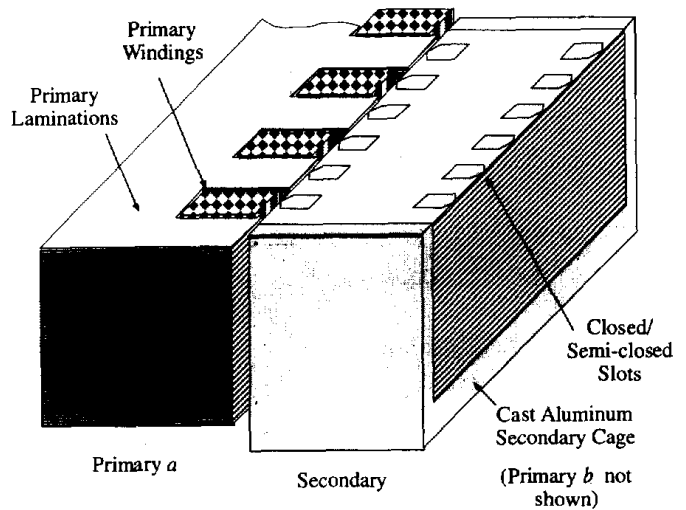

Fig. 1. Sectional view of a double-sided LFLIM

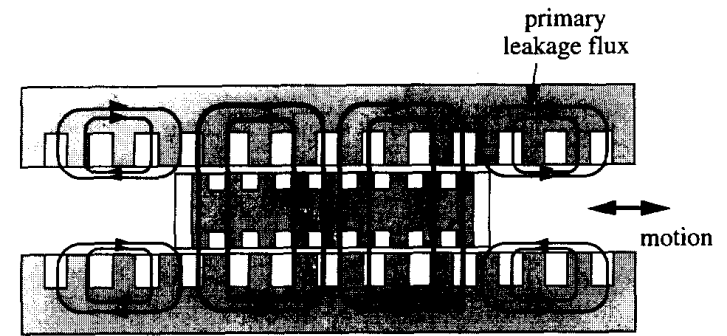

Fig. 2. Simplified magnetic flux paths in a straight double-sided LFLIM under balanced excitation such that zero net normal force is exerted on/by the secondary (a) and (b).

\section{LONGITUDINAL-FLUX LINEAR INDUCTION MACHINE (LFLIM) DESIGN}

Fig. 1 shows a cross-sectional view of a straight doublesided LFLIM with a cast aluminum secondary. The magnetic flux paths at one instant of time with a two-pole secondary and balanced normal forces are conceptually illustrated in Fig. 2 . Under this operating condition, no flux flows longitudinally within the secondary core, and thus the secondary core is not utilized.

An LFLIM with curved primaries under unbalanced excitation such that a net normal force is generated is shown in Fig. 3(a). Generation of the normal force requires that the difference between the air gap flux on each side flows longitudinally within the secondary core. The secondary core must be sized to accommodate this flux differential. For a given radius of curvature, the top speed at which steering can safely occur will be limited by the maximum normal force that can be generated. The secondary and primary core depths must thus be comparably sized. In an LFLIM, the required core depth is directly proportional to the pole pitch. Hence, a short pole pitch is desirable to minimize the secondary core and thus vehicle mass.

Design laws in induction machines favor a large pole pitch to air gap length ratio to maximize the magnetizing inductance. The minimum air gap length will be set by mechanical tolerances, which for assembly reasons will be large relative to rotating machine tolerances. Because of the inherently large leakage inductance due to the long-primary topology, a long pole pitch is preferred.

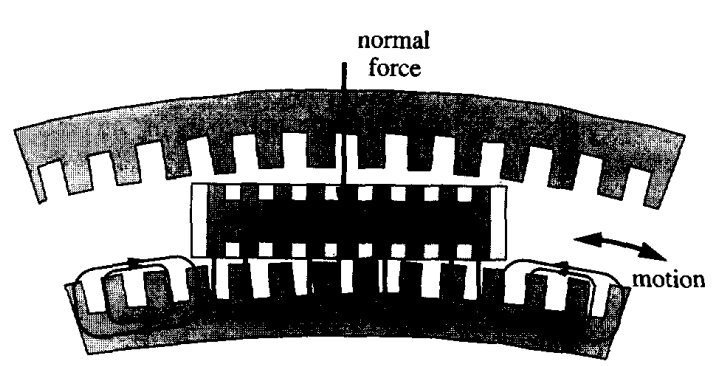

(a)

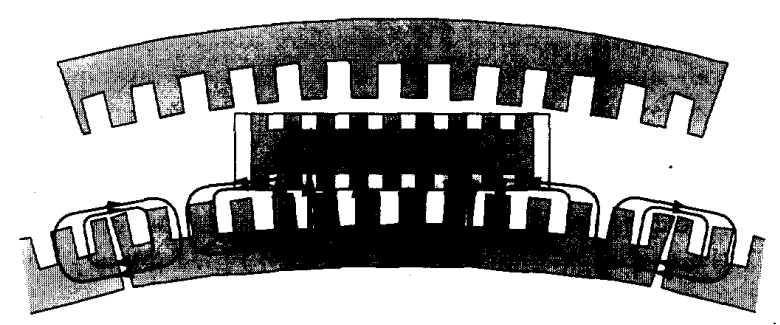

(b)

Fig. 3. Conceptual magnetic flux paths at two instants in time in a curvilinear LFLIM under unbalanced (i.e., single-sided) excitation generating the maximum possible net normal force for steering/centrifugal force compensation. The flux paths in (b) are altered from the ideal due to static end effects.

Two additional design complications are the presence of air gap chording and of static end effects. As illustrated in Fig. 3, the air gap length varies when a straight, rigid secondary is within a curved primary. With a nominal (minimum) air gap length of $1.5 \mathrm{~mm}$, a two-pole secondary with a $0.083-\mathrm{m}$ pole pitch in a $1-\mathrm{m}$ radius will experience an air gap variation from 1.5 to $\approx 5 \mathrm{~mm}$. Assuming a sinusoidally distributed primary winding and no saturation, the magnetizing inductance will be reduced by $\approx 30 \%$ compared to the constant $1.5-\mathrm{mm}$ gap length condition. Furthermore, the phases will be unbalanced and the air gap flux distribution will be concentrated near the secondary midpoint. With respect to normal forces, chording effects will thus reduce both the maximum possible magnitude and the ability to manipulate the moment. Short secondary lengths are thus favored to minimize chording effects.

Static end effects are present due to both magnetic and electric circuit discontinuities in the secondary. Although the flux paths are as desired at the instant illustrated in Fig. 3(a), the flux paths are less than ideal at the later instant illustrated in Fig. 3(b). Both the primary and secondary cores must accommodate the full flux per pole as shown, although the actual field patterns are considerably more complicated. These magnetic end effects significantly compromise the generation and control of the thrust and normal forces. The end effects increase drastically with pole numbers less than two and decrease with increasing pole numbers.

The end effects due to electric circuit discontinuities are similar to the magnetic end effects. The differences being that the electric currents are confined to the cage circuit and saturation does not occur. In consideration of both forms of end effects, a short pole pitch is favored for a given length secondary. 
Ultimately, a compromise between the competing design constraints acting to maximize the pole pitch to air gap ratio, minimize the secondary length, and to minimize static end effects suggests a secondary pole number of around two. The secondary and primary cores must then be oversized to accommodate the full flux per pole.

Note that during balanced operation within straight primaries, the secondary core acts merely as dead weight, significantly limiting the maximum achievable acceleration. If normal force production were not required, the secondary core could be entirely eliminated, drastically reducing the secondary mass with little or no reduction of thrust capability or efficiency.

Both magnetic and electric end effects are also present due to the finite length of the primary units. The end effects will be most severe when a secondary bridges two primary units. A reduction in both the thrust and normal forces can be expected. Since the interruption of normal force generation can be catastrophic during steering, the LFLIM primary must be a continuous unit along curves.

The construction of straight LFLIM primaries is not difficult. Low-cost notching dies are typically used to produce lamination segments of desired length with little material waste. The construction of curved primary laminations, however, requires more expensive custom dies and produces a potentially large amount of waste. Furthermore, each lamination design is generally applicable to only one intended radius of curvature. A complete transport system would thus require either numerous sets of different curved laminations or be restricted to a single radius of curvature in addition to the straight sections.

In summary, the LFLIM topology does not readily lend itself to a modular, curved, primary construction capable of both high acceleration and large normal force generation and control.

\section{TRANSVERSE-FluX LinEAR INDUCTION MACHINE (TFLIM) DESIGN}

By orienting the flux paths along a plane normal to the thrust vector, the TFLIM inherently overcomes many of the problems associated with the LFLIM. A secondary and one primary of a double-sided $E$-core TFLIM is illustrated in Fig. 4. Unlike the LFLIM, the laminations are stacked along the longitudinal axis with nonmagnetic spacers imbedded to form "lamination sets."

The main flux paths for the $E$-core and also a $C$-core TFLIM are illustrated in Fig. 5. To generate a net normal force, a flux differential must flow (vertically) through the secondary core. Even though the flux is confined to a transverse plane, longitudinally traveling MMF and air gap flux waves are still created. The secondary cage is more complicated than the LFLIM secondary cage, though induced currents still interact with the traveling wave to produce a longitudinal thrust vector. Whereas the LFLIM cage effectively contained a single electric circuit per side, the $E$-core TFLIM cage contains three circuits per side (two for the $C$-core). With the cast cages as shown, the individual circuits are highly coupled between sides in both machines.

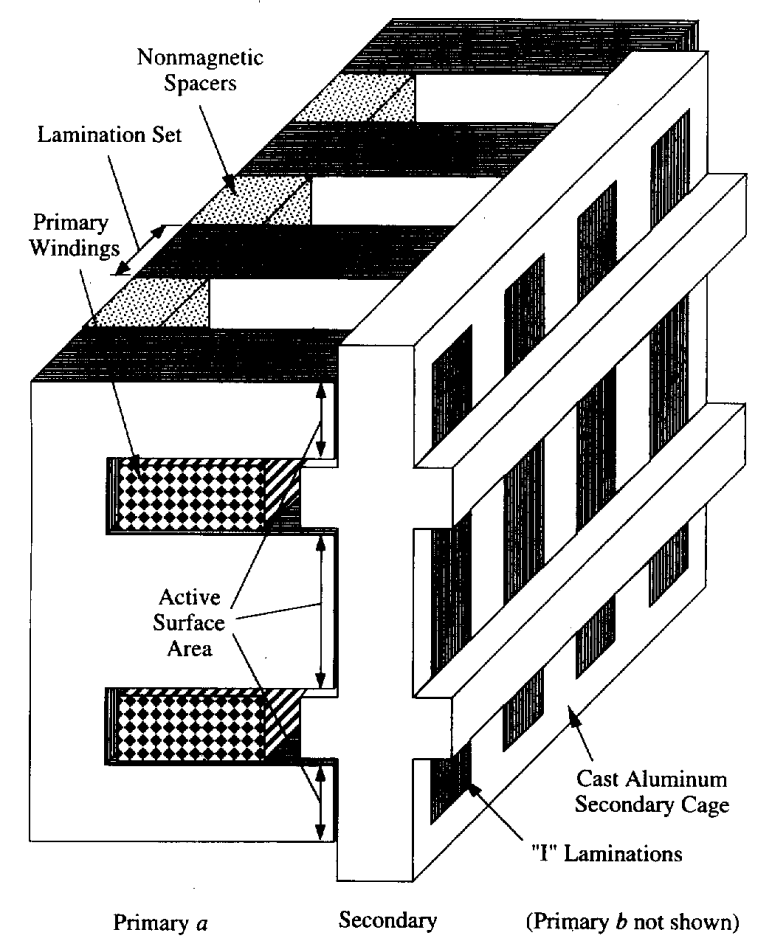

Fig. 4. Illustration of a double-sided E-core TFLIM.

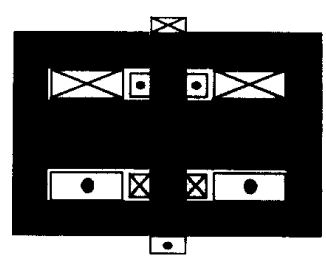

(a)

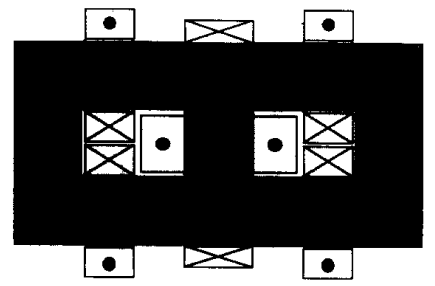

(b)
Fig. 5. End views illustrating the main flux paths and currents of (a) $E$-core and (b) $C$-core TFLIM's of the same magnetic loading and hence capable of achieving the same normal force.

An immediately appealing outcome of the transverse flux orientation is that the flux in each lamination set is selfcontained. Unlike the LFLIM, the core depth is thus independent of pole pitch. For a secondary of a given length, the required core depth is specified by the active surface area in the air gap of an TFLIM. With the magnetic and electric loadings limited by saturation and thermal bounds, respectively, the maximum thrust production is proportional to the active area. Hence, the TFLIM core depth is essentially thrust dependent, while the LFLIM core depth is velocity dependent.

It therefore follows that in regard to core mass, LFLIM's are favorable for short pole pitch, wide geometries, while TFLIM's are favorable for long pole pitch, narrow geometries. It was for this reason that the TFLIM received considerable attention for ground transport systems in the 1970's [10]-[12]. Prior to viable, variable frequency excitation, the long pole pitches necessary to achieve high velocities $(200-400 \mathrm{~km} / \mathrm{h})$ with only 50 or $60 \mathrm{~Hz}$ excitation led to massive cores in LFLIM's. With variable frequency excitation now both reliable 


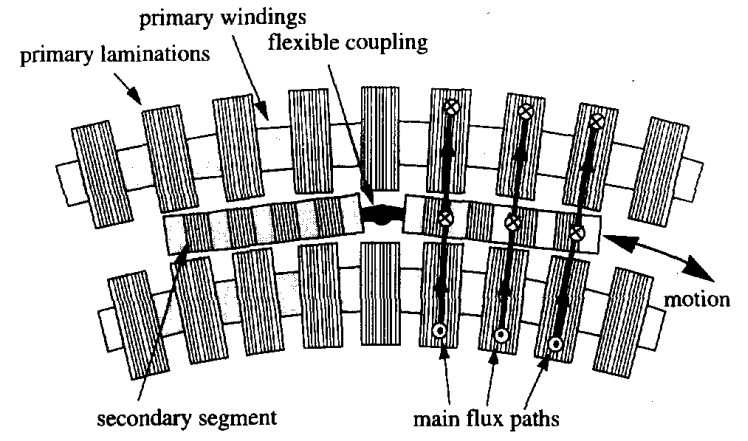

Fig. 6. Top view of a curvilinear TFLIM with an articulated secondary to reduce chording effects.

and economical, and a lower maximum velocity, the core depth/velocity relationship is of reduced significance to the material transport system in this study. The core depth/end effect relationship, however, is of considerable relevance.

The self-containment of the flux in the TFLIM also eliminates all magnetic end effects in both the primary and secondary. As shown in Fig. 6, the secondary can then be articulated without detrimental effects on the magnetic circuit, thereby reducing air gap chording and increasing the magnetizing inductance within curved primaries for the same pole pitch. Unlike the LFLIM, oversized cores are not required.

Unfortunately, electrical end effects still exist and are particularly troublesome in the secondary. As the secondary cage is shortened relative to the pole pitch, the effective secondary resistance increases and becomes asymmetrical. With respect to thrust production and efficiency, increases in the magnetizing inductance tend to be offset by the increased secondary resistance. Analysis and experimental results have shown that the machine performance deteriorates rapidly as segment lengths decrease below $\approx 1.5$ pole pitches, i.e., average thrust decreases while thrust pulsations at twice slip frequency increase in magnitude.

The TFLIM can be constructed using low-cost, standard $C$ or $E$-core transformer laminations to create virtually any radius of curvature. The corresponding transformer " $I$ " sections are used within the secondary. Cast epoxy can be used to retain the primary windings and provide support between the lamination sets. With the absence of magnetic end effects, the TFLIM lends itself to a highly modular construction.

Although Gramme-ring type windings concentrated over individual lamination sets were considered in [5], [10], the more conventional distributed primary windings as shown in Fig. 7 are favored, especially for the short pole pitches under consideration. Two influential factors are the fill factor of the lamination slot and the required spacing between lamination sets. Although the Gramme-ring winding will result in a higher fill factor, both the MMF fundamental and the winding utilization are lower. Furthermore, the distributed winding requires less space between lamination sets, and thus a higher magnetic loading can be achieved for the same MMF. Winding insertion and protection is somewhat complicated by the constricted end winding (i.e., lamination slot) window area.

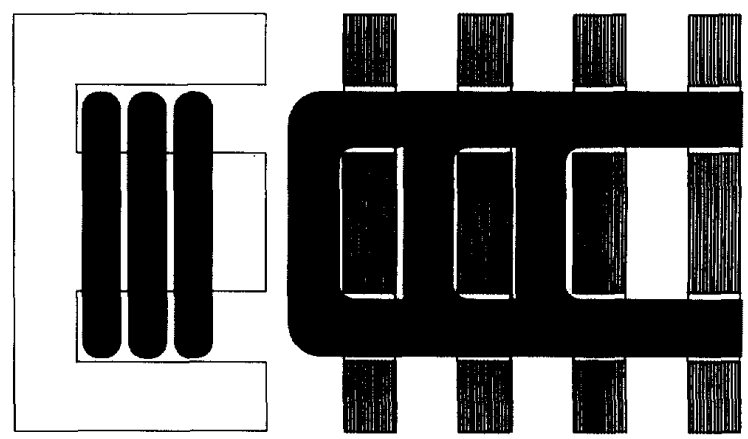

Fig. 7. Distributed primary windings in a $E$-core TFLIM.

Two immediately apparent drawbacks of the lamination orientation in the TFLIM are that only fully-open, unskewed slots are easily fabricated and that increased core loss is to be expected at high speeds. The orientation of the laminations is conducive to increased eddy currents at high excitation frequencies and velocities. The effect will be similar to dynamic end effects experienced in long-secondary topologies, albeit at a reduced level.

\section{COMPARISON OF LFLIM'S AND TFLIM'S}

A meaningful comparison between the two topologies can be formed on the basis of equal magnetic loadings and air gap lengths, where equal magnetic loading will be defined to imply equal air gap flux density and net active surface area (hence equal normal forces). The active surface widths are then also equal if the tooth/slot width ratios (i.e., with respect to the lamination set/spacer in the TFLIM) are constrained equal. Saturation will be neglected.

\section{A. Primary Magnetizing Inductance}

Under the above constraints, the TFLIM requires twice the MMF as the LFLIM, because the flux must cross two air gaps. With surface areas of one half the LFLIM surface area and assuming the same number of turns per pole, the magnetizing inductance of the TFLIM is one quarter that of the LFLIM. Alternatively, for the same flux linkage per pole, the TFLIM requires twice the number of turns. Although the magnetizing inductanc ss are then equal, the primary and effective secondary resist ses have also increased by a factor of four (assuming the sar window area). In both cases, the secondary time constant (: measure of the machine quality or Goodness [7]) has remained fixed.

\section{B. Primary Leakage Inductance}

In many aspects, the primary end winding and active regions of the TFLIM and LFLIM are reversed. In an LFLIM, the active region is enclosed within slots while the end region, which contains the net MMF per pole, is external to the lamination stack. In a TFLIM, the "end region" is contained within the slots of the individual laminations, while the "active region" is contained between laminations sets. The corresponding leakage flux paths for the $E$ and $C$ core TFLIM's are illustrated in Fig. 8. 


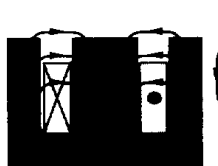

(a)

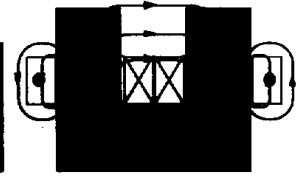

(b)

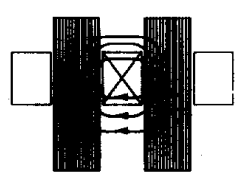

(c)
Fig. 8. Hlustration of primary slot leakage within individual laminations in (a) $E$-core and (b) $C$-core TFLIM's, and (c) between lamination sets (a) and (b).

With enclosed end windings carrying the net MMF per pole and inductance being related by the square, the effective end winding inductance in a TFLIM can be much greater than either the end winding or slot leakage inductance in an LFLIM. The end winding inductance, like the magnetizing inductance, will increase with pole pitch.

Comparing the $C$ and $E$-core TFLIM's, for the same net window, core, and active surface areas, the MMF requirements for the $E$ and $C$-core TFLIM's are identical. Since the $E$-core primary has twice the number of slots, each being half the width, but enclosing the same MMF as the $C$-core primary, a $4: 1$ ratio in slot leakage inductances for the internal winding sections can be expected. The additional external winding sections of the $C$-core will reduce the ratio somewhat.

The LFLIM can thus be expected to offer the lowest primary leakage inductance, followed by the $C$-core TFLIM, with the $E$-core TFLIM producing the highest leakage.

\section{Secondary Magnetic and Electric Circuits}

Fig. 9(a) illustrates a single slot pitch section of each secondary assuming equal tooth/slot width ratios. The volumes indicated by the dashed lines are the effective cores required for equivalent maximum normal force generation with a pole pitch of $0.125 \mathrm{~m}$. For the given dimensions, the $E$-core TFLIM offers the highest normal force per unit core mass. The $C$ core TFLIM will always offer a lower normal force to mass ratio than the $E$-core. The normal force to mass ratio for the LFLIM will decrease with increasing pole pitch, thus favoring a shorter pole pitch.

With the above constraints, the electrical circuits of the TFLIM secondary are equivalent to sectioning the LFLIM secondary circuit into two or three smaller circuits as illustrated in Fig. 9(b). For the same magnetic loading, equal thrust production requires equal current loadings, i.e., $i_{L}=i_{C}=$ $i_{E 1}=i_{E 2}$. Thus, equal thrust at the same slip frequency requires that the secondary resistances are $r_{C}=r_{E 1}=r_{L} / 2$, and $r_{E 2}=r_{L} / 4$. Of course, the actual current loops are distributed over a full pole pitch and thus are considerably more complicated, especially in the presence of end effects. But nonetheless, the TFLIM secondary requires considerably more aluminum for the same slip yielding peak thrust.

If the magnetizing inductances of the three machines are equal, the $E$-core may also offer the highest thrust/amp per unit core mass. However, the slip yielding peak thrust may be considerably higher, resulting in higher losses.

In many ways, at least with respect to the secondary, the comparison between the LFLIM and the two TFLIM's is similar to comparing one large induction machine to two or

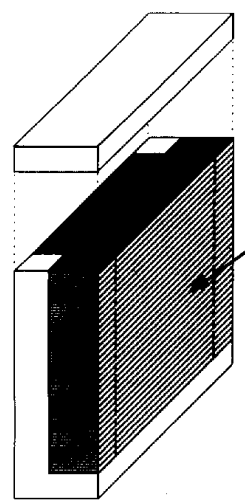

LFLIM

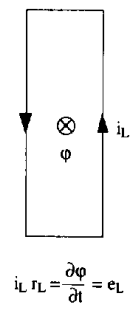

LFLIM

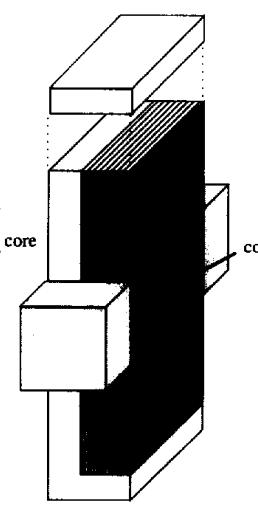

C-core TFLIM

(a)

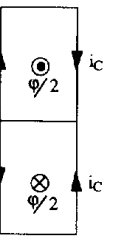

$i_{C} r_{C}=\frac{\partial \varphi / 2}{\partial t}=\frac{e_{L}}{2}$

C-core TFLIM

(b)

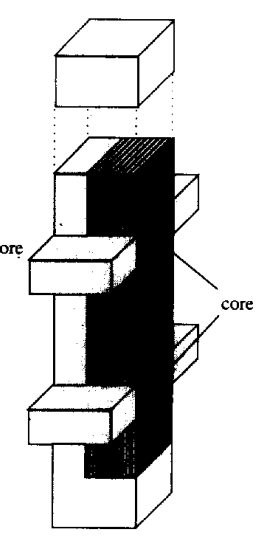

E-core TFLIM

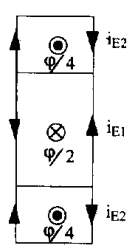

$i_{E I} r_{E I}=\frac{\partial \varphi / 2}{\partial t}=\frac{e_{L}}{2}$

$\mathrm{i}_{\mathrm{E} 2} \mathrm{I}_{\mathrm{E} Z}=\frac{\partial \varphi / 4}{\partial \mathrm{t}}=\frac{\mathrm{e}_{\mathrm{L}}}{4}$

E-core TFLIM
Fig. 9. Comparison between the three secondary topologies based upon a single slot pitch with equivalent magnetic loadings. Laminations are stacked as shown with aluminum conductors represented by the lighter shading. Dashed lines separate the effective tooth and core volumes. $j$ and $e_{L}$ are the secondary flux and induced voltage, respectively, for the LFLIM.

three smaller machines with the same combined ratings. As is well known, scaling laws favor the larger machine.

In summary, the relative merits of the LFLIM can be generalized by recognizing that it offers the overall best machine quality (i.e., higher magnetizing/leakage inductance ratio, larger secondary time constant, etc.) especially for straight primaries in the factory scaled system. Distinct disadvantages include the excessive secondary mass, the existence of both magnetic and electrical static end effects, and an inherent difficulty in constructing curved modular primaries.

The TFLIM offers the advantages of having no magnetic end effects, flexibility through the use of low-cost, standard transformer laminations to construct secondaries and primaries of any radius of curvature, and minimal secondary core iron. A distinct disadvantage is the low magnetizing/leakage inductance ratio. Unlike the LFLIM, it is ideally suited for modular curved primaries.

\section{A HYBRID SYSTEM UTILIZING BOTH LONGITUDINAL AND TRANSVERSE-FLUX TOPOLOGIES}

The best attributes of both the LFLIM and the TFLIM can be obtained via a hybrid system topology that operates as an LFLIM within straight primary units, and a TFLIM within curvilinear primary units. The secondary and TFLIM primary 


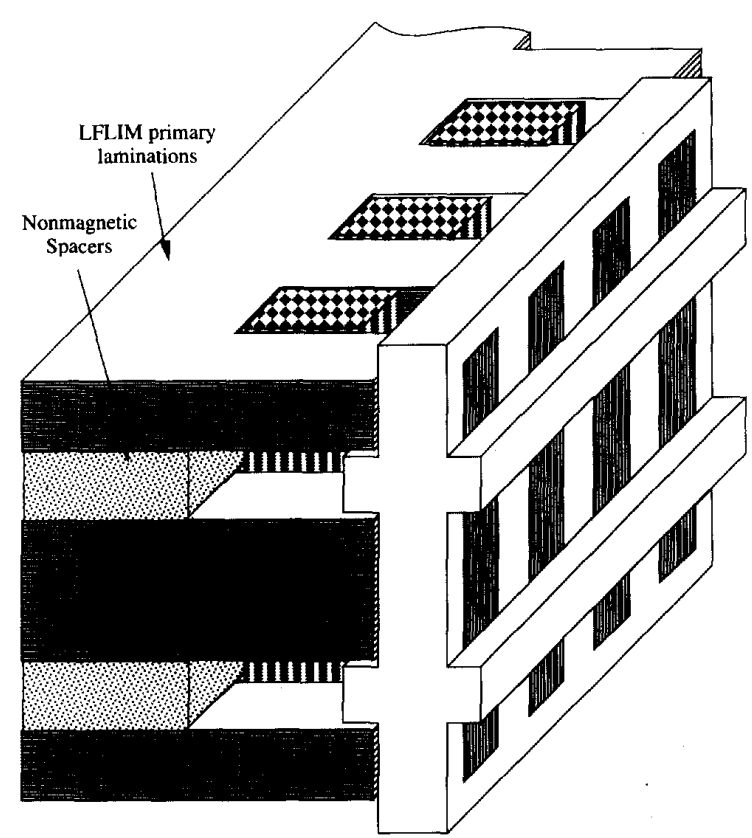

Fig. 10. Sectional view of a hybrid LFLIM/E-core TFLIM.

of the hybrid system are identical to the $E$ (or $C$-core) TFLIM in Fig. 4. The LFLIM primary must be altered to accommodate the external longitudinal bars of the secondary cage as shown in Fig. 10 for an $E$-core system.

Compared to the original LFLIM in Fig. 1, two major penalties imparted on the hybrid LFLIM design are an increased primary slot depth and an additional inactive region of the primary windings. The leakage inductance and copper losses will then be larger. Two additional penalties are the open secondary slots and the nonideal orientation of the secondary laminations inherited from the TFLIM secondary.

The penalties of the hybrid LFLIM are outweighed by two additional benefits unique to the hybrid system. Because the TFLIM secondary core does not provide a longitudinal flux path (unlike the LFLIM core in Fig. 1), all magnetic flux is forced to cross both air gaps when operating as an LFLIM as illustrated in Fig. 11. Hence, at least to a good approximation, the normal forces will nearly always be balanced with reasonably balanced excitation and secondary alignment.

Although magnetic end effects will still exist in the LFLIM primaries, they are eliminated from the secondary. The oversized secondary core required by the original LFLIM in Fig. 1 is replaced by the significantly smaller core of the TFLIM. When operating as a LFLIM, the secondary cage links the entire flux in one circuit, not two or three. Although the external longitudinal bars of the TFLIM secondary cage are not then utilized, they add structural integrity with little additional mass.

An unresolved issue of the hybrid topology involves the transition region in which the secondary is crossing between the LFLIM and the TFLIM primaries (and vice versa). The dynamics will undoubtedly result in an interruption of thrust

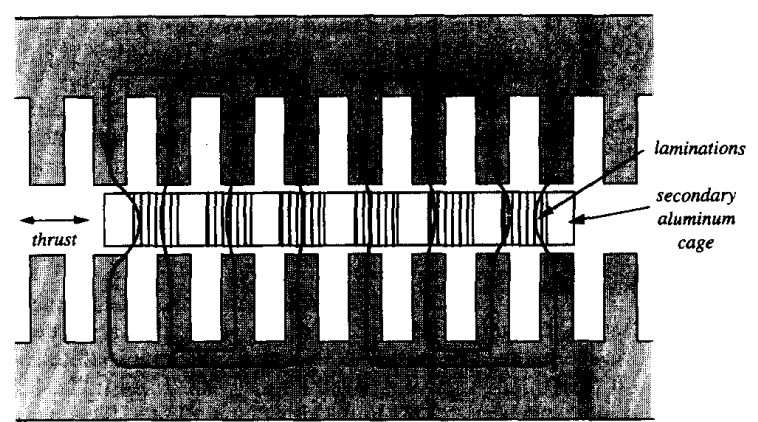

Fig. 11. Illustration of the magnetic flux paths in a hybrid system consisting of a TFLIM secondary within LFLIM primaries.

production, along with a time constant associated with the generation of normal forces.

\section{CONCLUSION}

This paper attempted to present the differences between the TFLIM and LFLIM in a new light. The performance specifications set for the transport system place severe and unique demands upon the linear machine design. Even though the TFLIM is not a "good" machine in general, it does have some attractive attributes that can be utilized in the proposed material transport system. A hybrid system topology appears to combine the best attributes of both the TFLIM and the LFLIM with additional advantages not obtainable with systems based solely upon either of the two topologies.

\section{REFERENCES}

[1] P. L. Jansen, L. J. Li, B. B. Werner, and R. D. Lorenz, "An integrated vehicle and electromagnetic propulsion unit for a high-speed material transport system," in Proc. IEEE Ind. Applicat. Soc. Annu. Meeting, Houston, TX, Oct. 1992.

[2] N. A. Duffie, R. D. Lorenz and J. L. Sanders, "High-performance LIMbased material transfer," in Proc. NSF Design and Manufacturing Syst. Conf., Atlanta, GA, Jan. 8-10, 1992.

[3] G. H. Abdou and S. A. Sherif, "Theoretical and experimental design of LIM in automated manufacturing systems," IEEE Trans. Ind. Applicat., vol. 27, no. 2, pp. 286-293, Mar./Apr. 1991.

[4] G. W. McLean, "Review of recent progress in linear motors," IEE Proc., vol. 135 , pt. B, no. 6 , pp. $380-416$, Nov. 1988.

[5] S. A. Nasar and I. Boldea, Linear Motion Electric Machines. New York: Wiley, 1976

[6] E. M. Freeman and D. A. Lowther, "Normal force in single-sided linear induction motors," Proc. IEE, vol. 120, no. 12, pp. 1499-1506, Dec. 1973.

[7] E. R. Laithwaite, Induction Machines for Special Purposes. London: George Newnes Ltd., 1966.

[8] P. L. Jansen, R. D. Lorenz and D. W. Novotny, "Observer-based direct field orientation: Analysis and comparison of alternative methods," IEEE Trans. Ind. Applicat., vol. 30, no. 4, pp. 945-953, July/Aug. 1994.

[9] P. L. Jansen and R. D. Lorenz, "Accuracy limitations of velocity and flux estimation in direct field oriented induction machines," in Proc. EPE Conf., Brighton, UK, Sept. 1993.

[10] E. R. Laithwaite, J. F. Eastham, H. R. Bolton and T. G. Fellows, "Linear motors with transverse flux," Proc. IEE, vol. 118, no. 12, pp. 1761-1767, Dec. 1971.

[11] J. F Eastham and E. R. Laithwaite, "Linear-motor topology," Proc. IEE, vol. 120, no. 3, pp. 337-343, Mar. 1973.

[12] J. S. Chahal, "Some aspects of a transverse flux linear induction motor design, suitable for high speed applications," in Conf. Linear Electric Machines, 1974, pp. 161-166. 


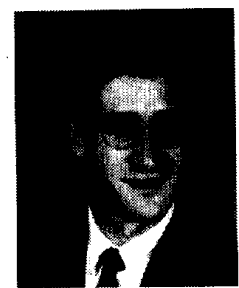

Patrick L. Jansen received the B.S., M.S., and Ph.D. degrees in electrical engineering from the University of Wisconsin, Madison, in 1985, 1987, and 1993, respectively. His research and dissertation at the University of Wisconsin focused on the integration of electric machine design with transducerless position and velocity estimation, observer-based direct field oriented ac drives and linear induction machine design for high-speed material transport systems.

From 1987 to 1989 , he was a development engineer at Machine Dynamics Pty. Ltd., Victoria, Australia, and later an experimental scientist at the Commonwealth Scientific and Industrial Research Organization (CSIRO), Division of Applied Physics, NSW, Australia. He is presently a senior engineer at MK Rail Corporation, Boise, ID, involved in the development of ac propulsion systems for locomotives. $\mathrm{He}$ is an Affiliate Assistant Professor in the Department of Electrical Engineering at the University of Idaho, Moscow. He has two patents pending.

Dr. Jansen has received two Prize Paper Awards from the IEEE Industry Applications Society Industrial Drives Committee.

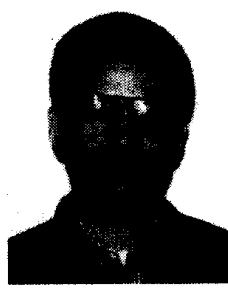

Long-Jang $\mathrm{Li}$ received the B.S. degree from $\mathrm{Na}$ tional Cheng Kung University, Taiwan, R.O.C., in 1981 , and the M.S. degree from the University of Wisconsin, Madison in 1986, both in mechanical engineering.

$\mathrm{He}$ is currently a Ph.D. candidate in mechanical engineering at the University of Wisconsin, Madison. His current research is focused on constructing a multi-unit subsystem prototype for the TFLIMbased material/tool transport system to demonstrate the capability of high-performance acceleration and electromagnetic steering.

Robert D. Lorenz (S'83-M'84-SM'91), for photograph and biography, please see p. 801 of this Transactions. 